\title{
Incontinência urinária feminina: uma revisão bibliográfica
}

\author{
Female urinary incontinence: a literature review \\ Incontinencia urinaria femenina: una revisión bibliográfica
}

Paula Barros Perreira ${ }^{1 *}$, Luis Alberto Leon Camac ${ }^{1}$, Francisca Amanda de Souza Mesquita ${ }^{1}$, Mario Clodoaldo Batista da Costa².

\begin{abstract}
RESUMO
Objetivo:Descrever o impacto da incontinência urinária feminina narotina diária.Métodos:Foi realizado uma revisão bibliográfica atualizada sobre incontinência urinaria feminina direcionada aos aspectos de qualidade de vida obtida da pesquisa na base de dados eletrônicas Scielo, Lilacs, Medline e PubMed.Resultados:O motivo das mulheres optarem por não buscar tratamento pode ser atribuído à desesperança, à depressão e ao constrangimento de ter que compartilhar sua condição com outros indivíduos. Diagnósticos correto é importante na avaliação e no tratamento de mulheres com IU, assim como na determinação do efeito sobre a Qualidade de Vida (QV) da mulher. Diversas formas de tratamento podem ser aplicadas para o tratamento das IU, incluindo medicação, exercícios que sejam específicos, algumas técnicas de estimulação dentre muitas outras.Conclusão: Intervenções e acompanhamento quanto à prevenção da Incontinência urinária desde o início e manter o fortalecimento da musculatura pélvica devem ser voltadas a mulheres que apresentem sintomas mesmo que sejam iniciais afim de conseguir uma eficiência no tratamento.
\end{abstract}

Palavras-Chave: Incontinência Urinária, tratamento, Qualidade de vida.

\begin{abstract}
Objective: To describe the impact of female urinary incontinence on narotina diaria. Methods: We carried out anupdated bibliography icreview on female urinary incontinence directed to aspects of quality life obtained from there search in the data base Scielo, Lilacs, Medline and, PubMed. Results: The reason women choose not to seek treatment an beat tribute to hopelessness, depression and the embarrassment fhaving to share their condition with other individuals. Correct diagnosis is important in the evaluation and treatment of women with UI, as well as in determining the effect on the Quality of Life (QoL) of the woman. Several forms of treatment can be applied for the treatment of UI, including medication, exercises that are specific, some stimulation techniques among many others. Conclusion: Intervention sand follow-up on the prevention of urinary incontinence from the beginning and maintaining the strengthening of the pelvic muscles should be targeted at women who present symptoms evenif they are initial in order to achieve treatment efficiency.
\end{abstract}

Key words: Urinaryincontinence, treatment, Qualityoflife.

\section{RESUMEN}

Objetivo:Describir el impacto de la incontinencia urinaria femenina en la rutinadiaria.Métodos:Fue realizado una revisión bibliográfica relacionada a incontinencia urinaria femenina orientada a los aspectos de calidad de vida obtenida de la base de datos de Scielo, Lilacs, Medline y PubMed.Resultados: El motivo de las mujeres optar por no buscar tratamiento puede ser atribuido a la desesperanza , a la depresión ya la restricción

\footnotetext{
1Universidade Federal do Amazonas (UFAM),Manaus -AM. *E-mail: paula_barros_p@hotmail.com

2Universidade Estadual do Amazonas (UEA), Manaus-AM.
} 
de tener que compartir su condición con otros individuos. Para realizar el diagnóstico correcto es importante la evaluación y el tratamiento de las mujeres con IU, así como en la determinación sobre la calidad de vida (QV) de la mujer. Las diversas formas de tratamiento puden ser aplicar para la terapia de las IU, incluyendo medicación, ejercicios específicos de fisioterapia del piso pelvico, algunas técnicas de estimulación entre muchas otras.Conclusión:Debe realizarse Intervenciones y seguimiento a fin de prevenir la incontinencia urinaria desde el principio, acompañadodel fortalecimiento de la musculatura del piso pélvicodirigida a las mujeres que presenten síntomas aunque sean iniciales, a fin de lograr un tratamiento eficaz.

Palabras clave: Incontinencia Urinaria, tratamiento, Calidad de vida.

\section{INTRODUÇÃO}

A incontinência urinária (IU)acomete milhares de pessoas de todas a faixa etária de idades, sendo com maior frequência as do sexo feminino, afetando diretamente a qualidade de suas vidas, além disso pode afetar mulheres de todas as idades, influenciar a saúde uroginecológica e a Qualidade de Vida (QV) da mulher e família (CORREIA S, et al., 2009).

A IU é definida pela International Continence Society (ICS) como qualquer queixa de perda de urina, independentemente do grau de desconforto social ou higiênico que cause, avançando em média $14 \%$ a $57 \%$ das mulheres com idade entre 20 e 89 anos (RINCON, 2015). O Brasil possui uma população que equivale a cerca de 190 milhões de habitantes, onde aproximadamente 56\% são mulheres, dentre elas um terço desta população é acometida pela IU (VIANA SBP, et al., 2012).

A IU é uma patologia conhecida pelo seu grau de negatividade ocasionado pelos diferentes domínios da vida da mulher, não só a nível físico mas também a nível psíquico, emocional e social, com custos substanciais a nível econômico (BOTLERO $\mathrm{R}$ et al.,2008). Considera como um problema multifatorial, o que gera consequências de nível físico e bem estar (higiene, mau odor e vestuário molhado); psicológico (diminuição da auto estima, aumento de estresse, oscilações de humor, sintomas de depressão); sociocultural (isolamento social), profissional (absentismo laboral, menor rendimento na produtividade); e económico-financeiro (despesa acrescida em roupa interior, fraldas, entre outros), e estas consequências conduzem a uma pior qualidade de vida das mulheres nos diferentes grupos etários (BASAK, T, 2013).

Segundo Botelho F et al. (2007), a IU, propicia a perda de urina por esforço no ato de - esforço, tosse ou espirro; de urgência - quando a vontade de urinar é súbita e incontrolável; e mista - quando existem sinais e sintomas dos dois tipos mencionados.

Melo BES et al, (2012), classificaa IU em três momentos os principais: (1) a Incontinência Urinária de Esforço (IUE), quando ocorre perda de urina durante algum esforço que aumente a pressão intra-abdominal, como tosse, espirro ou exercícios físicos; (2) a urge-incontinência ou Incontinência Urinária de Urgência (IUU), caracterizada pela perca de urina seguida por forte sensação de urgência em urinar; e a Incontinência Urinária Mista (IUM), quando há queixa de perca de urina associada à urgência e também a esforços.

Diversos fatores de risco podem estar interligados ao aparecimento de sintomas, entre eles, o próprio envelhecimento que ocorre natural nas fibras musculares, a redução da função ovariana após a menopausa, obesidade, gravidez e múltiplos partos vaginais (SOUSA JG et al., 2011).

A forma de armazenar e esvaziar ocorre em processo fisiologicamente complexo e, para que possa ter um bom funcionamento, necessita que diferentes músculos, nervos parassimpáticos, simpáticos, somáticos e sensoriais trabalhem em conjunto, e se houver qualquer deficiência das citadas acima pode desenvolvera IU (GIRÃO MJBC, et al., 1997).

O exame físico é uma rotina na etapa de investigação ginecológica da mulher incontinente, uma vez que tenta reproduzir e caracterizar a incontinência, a fim de excluir distúrbios neurológicos, avaliando o suporte pélvico e excluir outras enfermidades pélvicas (FINE PFM, 2004). 
Já o exame clínico da paciente com hipótese de bexiga hiperativa é realizado em posição ginecológica, se de preferência com a bexiga cheia. Outros testes devem ser realizados para um melhor diagnósticos dentre eles encontra-se o exame de urina, que busca analisar o sedimento, já a urocultura, considera-se obrigatória em pacientes com queixas uroginecológicas. São necessários, haja vista que infecções urinárias podem ser as causas dos sintomas e, com grande probabilidade, de possuir manipulação instrumental do trato urinário (FELDNER PC JR, 2006).

Os exames de imagem como a ultrassonografia, busca avaliar o resíduo miccional, procura detectar tumores vesicais e distorções na parede vesical por massas pélvicas, e ter uma avaliação da vascularização dos tecidos periuretrais por meio do efeito Doppler e da musculatura do assoalho pélvico, afim de avaliar a hipermobilidade e a posição do colo vesical.O tratamento da IU constitui por duas modalidades: conservador e cirúrgico. O tratamento cirúrgico não é efetivo em todos os casos, além do que ocorre recidiva dos sintomas antes de cinco anos (BERNARDES NO, et al., 2000).

Mesquita LA et al, (2010) relata que a terapia comportamental é uma opção primária para começar um tratamento, inicia-se com a observação e alterações da relação do sintoma da paciente e seu ambiente e assim modifica maus hábitos miccionais. Essa terapia auxilia a paciente a compreender a necessidade da reeducação do hábito urinário, exercícios de consciência e fortalecimento dos músculos do assoalho pélvico, orientar estratégias para um melhor controle do desejo miccional e uma dieta ingesta hídrica (MESQUITA LA et al, 2010). Já na visão de Rios LA; Gomes HP, (2010), o método de Slings minimiza uma grande cirurgia para uma micro-invasiva, reduzindo não só o tempo cirúrgico, mas melhorando a hospitalização, o desconforto pós-operatório e tempo de recuperação. O uso de sling sintético colocado sob a uretra média e revolucionou o tratamento da IUE.

A utilização de medicamentos no tratamento de interface é baseada na existência de ceptoresneurorrecolinérgico, adrenérgico efaadrenérgicos que produzem contração ou relaxamento vesicoureteral dependendo maço farmacológicas administrado (CALVO A, 2002).

O objetivo foi discorrer e abordar sobre a incontinência urinária, e o que pode afetar na qualidade de vida destas mulheres.

\section{MÉTODOS}

O presente trabalho caracteriza-se como uma revisão bibliográfica. A coleta de dados ocorreu através do levantamento de publicações científicas sobre IU em mulheres. A revisão foi realizada a partir das bases de dados eletrônicos tais como: Scielo, Lilacs, Medline, PubMed. Nas bases, foram utilizados os descritores Incontinência Urinária, tratamento, Qualidade de vida. Os artigos foram selecionados segundo os seguintes critérios de inclusão: estar redigido em língua inglesa, espanhola ou portuguesa e abordar temas relacionados à IU feminina. Excluíram-se os artigos que enfatizavam IU masculina, infantil, em idosos.

\section{RESULTADOS E DISCUSSÃO}

A incontinência urinária é resultante de alterações anatômicas e funcionais. É sugerido que devido as diferenças anatômicas, a IUE atinja predominantemente as mulheres. As diferenças orificiais do sistema perineal ocasionam maior debilidade ao períneo feminino associado a um menor comprimento da uretra parecem contribuir para uma maior prevalência no sexo feminino (CONNOR S, 2004).

O exame físico é muito freqüente e parte da rotina de investigação ginecológica da mulher que apresenta os sintomas da incontinência, podendo assim reproduzir e caracterizar qual é a incontinência, e com isso eliminar distúrbios neurológicos, finalizando com a exclusão de outras e possíveis enfermidades pélvicas (FINE PFM,2004).

Já o exame clínico busca ser realizado com a paciente onde o diagnóstico de bexiga hiperativa deve ser realizado em posição ginecológica, preferencialmente com a bexiga cheia. Já outros testes possibilitam outras 
observações, um deles é o exame de urina, que a partir de sua coleta pode-se analisar os sedimentos presentes, já a urocultura, torna-se obrigatória quando há queixas uroginecológicas. Exames que se faz necessário, haja visto que as infecções urinárias podem ser causa dos sintomas, sendo assim existirá manipulação instrumental do trato urinário (FELDNER PC JR, 2006).

Exames de imagens como a ultrassonografia, buscam dar valor ao resíduo miccional, identificar e detectar tumores vesicais e as distorções que provocam na parede vesical por massas pélvicas, avaliando a vascularização dos tecidos periuretrais por meio do Doppler e da musculatura do assoalho pélvico, considerando a hipermobilidade e a posição do colo vesical. O tratamento da IU por ser diferenciado por duas modalidades: o conservador e o cirúrgico, sendo este último não efetivo em todos os casos, além do que ocorre reincidência dos sintomas antes de cinco anos (BERNADES NO,2000).

A terapia comportamental é sempre uma primeira opção para começar um tratamento, inicia-se observando as alterações da relação do sintoma da paciente e seu ambiente e assim modifica maus hábitos miccionais. Esta terapia ajuda na compreensão da paciente em reeducar seu hábito urinário, exercícios de consciência e fortalecimento dos músculos do assoalho pélvico, orientar estratégias para um melhor controle do desejo miccional e uma dieta ingesta hídrica (MESQUITA LA, et al.,2010).

O tratamento conservador mais dinâmico é a fisioterapia do assoalho pélvico, que proporicona várias técnicas como a terapia comportamental, cinesioterapia e eletroterapia, que estabelece motivação ao paciente, sendo uma importante parte para o sucesso do tratamento. $O$ tratamento da IUE pode ser conscientização e conhecimento da região perineal, fortalecimento dos músculos do assoalho pélvico (cinesioterapia), cones vaginais, biofeedback e a eletroestimulação (SANTOS PFD, 2010).

Rios LA; Gomes HP, 2010 diz que que os Slings propostos em meados do século XX, popularizaram-se no meio da urologia, após modificações técnicas introduzidas por McGuire. No final de 1990, Ulmsten propôs o uso de sling sintético colocado sob a uretra média e revolucionou o tratamento da IUE. Anteriormente utilizado apenas para casos de IU severa e por lesão esfincteriana, os slings passaram a ser utilizados para todos os tipos de IUE com resultados excelentes e duráveis. Introdução de sling sintético tornou a cirurgia mais simples e mais rápida, com uniformidade de resultados ao redor do globo.

A utilização de medicamentos no tratamento de interface é baseada na existência de ceptoresneurorrecolinérgico, adrenérgico efaadrenérgicos, que produzem contração ou relaxamento vesicoureteral dependendo maço farmacológicas administrado (CALVO A, 2002).

A idade pode ser considerada como um dos principais fatores de risco, pois sua prevalência IU tem aumento relevante após a menopausa (HIGA R, et al., 2008), o abaixamento do estrogênio sérico em mulheres nessa faixa etária. Os baixos níveis desse hormônio acarretam atrofia da musculatura uretral e facilitam com isso desenvolve a IU. Estes distúrbios urinários em mulheres de maior idade podem se dá pelo fato da capacidade menor de sua bexiga, passando de 500 a $600 \mathrm{ml}$ para 250 a $300 \mathrm{ml}$, o que força mais a alta freqüência urinária e da noctúria (PENN C, 1996).

Já a obesidade é um dos fatores que pode ter contribuição direta para o desenvolvimento da IU. Estimase que 46 a $67 \%$ das mulheres obesas apresentam IU (GERTEN KA, 2007). Segundo revisão realizada por Subak LL et al, (2009), estudos epidemiológicos mostraram que a obesidade é um forte fator de risco independente para a prevalência e incidência da IU e há um efeito dose-resposta do peso sobre a IU, sendo que o aumento de 5 unidades no IMC está associado a um aumento de cerca de $20 \%$ a $70 \%$ no risco de IU. No estudo de Brown JS et al, (1996), mulheres que apresentam IMC acima de $30 \mathrm{Kg} / \mathrm{m}^{2}$ têm um aumento do risco de IU em $40 \%$ quando comparadas a mulheres não-obesas (IMC $\left.<25 \mathrm{Kg} / \mathrm{m}^{2}\right)$, demonstrando também uma correlação positiva entre a IU e a obesidade.Pelo mesmo motivo, HAN MO et al, (2005), afirmaram que a probabilidade da IU por esforço em mulheres aumenta com o aumento da circunferência da cintura.

De acordo com Rasia J (2006), a menopausa é considerada um evento único que marca a transição do período reprodutivo para o não-reprodutivo. A menopausa natural é definida pela Organização Mundial de Saúde 9 como sendo 12 meses consecutivos de amenorreia, sem outra causa patológica ou psicológica. 
A estática pélvica afeta as condições hormonais durante a menopausa. O hipoestrogenismo na pósmenopausa predispõe a mulher à IU (BUTLER RN, 1999), desenvolvendo o aumento da frequência, urgência e disúria).

As cirurgias ginecológicas seguindo os autores Penn C e Brown JS, (1996), observaram achados de significância entre a histerectomia e a IU. Uma vez que a excisão ou o prolápso do útero compromete as funções do assoalho pélvico, sendo que este órgão suporta parte deste assoalho a sua remoção causando danos nas estruturas que sustenta a bexiga e a uretra. Em outro dois estudos de revisão bibliográfica, observou-se resultados divergentes, pois, um relatou que a histerectomia aumenta a prevalência IU e outro, não concluiu crescimento significativo de IU após a retirada do útero (PENN C, 1996).

As doenças crônicas: tais como a diabetes e doenças neurológicas são causas de risco para IU. Essa associação entre diabetes e a IU mostraram vulnerabilidade do assoalho pélvico com a mudança do tecido biológico e da inervação do músculo pélvico ou devido lesões nas inervações neuropáticas autônomas da bexiga ou pelo fato da freqüência urinária causada pela hiperglicemia decorrente do aumento do volume urinário (BROWN JS, 1996).

Fatores hereditários entre raça branca e de raças negras entre as mulheres, propuseram na observação do estudo que mulheres de raça branca têm maior prevalência, supondo que a causa provável estejam os determinantes genéticos, diferenças anatômicas ou até na resistência da uretra e nas estruturas de suporte do assoalho pélvico que protegem as mulheres negras da IU (BROWN JS, 1996). No Brasil, não encontraram diferença no risco de IU em relação à raça e, consideraram a possibilidade de que parte das mulheres da amostra estudada poderia ter traços de herança negra (ELIA G, 2002).

Uso de drogas nas medicações é fator que contribui para a IU transitória. Sendo que alguns medicamentos tendem a aumentar a freqüência e a urgência urinária (BUTLER RN, 1999).

É valido sempre avaliar o impacto ter a percepção da qualidade de vida em mulheres com IU. Diante de estudos concluiu-se que as mulheres com IU frequentemente apresentam uma diminuição da sua qualidade de vida (CORREIA S, et al., 2009; LASSERRE et al., 2009; BASAK T, et al., 2013).

Apesar de afetar na qualidade de vida, as taxas de procura por tratamento entre as incontinentes variam consideravelmente (SILVA L; LOPES MHBM, 2009).

O motivo das mulheres optarem por não buscar tratamento, mantendo a situação de sofrimento e a necessidade de constantes adaptações, pode ser atribuído à desesperança, à depressão e ao constrangimento de ter que compartilhar sua condição com outros indivíduos. O processo de negação e de evitar admitir a condição levam as sofredoras a um desgaste físico e emocional acentuado (NICOLSON P et al, 2008). Muitas das vezes estas mulheres apresentam-se deprimidas, psicologicamente estressadas, com diversos distúrbios sendo eles emocionam e sociais, causando até um isolamento (SESHAN V, 2014).

O tratamento multidisciplinar é acentuado, uma vez que cada profissional de saúde - médico, psicólogo e fisioterapeuta deverá realizar sua abordagem de forma integrada com os demais (TORREALBA FCM; OLIVEIRA CR, 2010). A ICS recomenda que o tratamento conservador seja acatado como primeira opção de intervenção, objetivando o aumento da força e a correta ativação da musculatura do assoalho pélvico (PEREIRA VS, et al., 2012).

Já o tratamento conservador fisioterapêutico da IU é voltado para o trabalho dos músculos pélvicos nas incontinências de esforço, de urgência e mistas. A fisioterapia proporciona recurso terapêutico eficiente, sem incômodo ou risco, compatível com outros recursos de tratamento. Envolve atividade específica de treino de percepção corporal e de normalização do tônus dos músculos pélvicos (VIANA SBP, et al., 2012). Pode utilizase de exercícios que estejam associados, ou não, ao uso de cones vaginais, biofeedback e eletroestimulação direta por corrente elétrica (BOTELHO F, 2007).

Em geral, as mulheres com IUM tendem a ser um pouco mais velhas que as mulheres com os outros tipos de IU (MINASSIAN VA, et al., 2013).

REAS/EJCH |Vol.11(14) | e1343 | DOI: https://doi.org/10.25248/reas.e1343.2019 Página 5 de 7 
Segundo Higa R, et al, 2008 quando se trata de faixa etária existe uma aceitação de um ser um fator de risco considerável pois a prevalência da IU aparece de forma frequente no período da menopausa, possibilitando um decréscimo do estrogênio sérico em mulheres. Assim também como níveis inferiores de hormônios o que pode desenvolver atrofia da muscular uretral e facilitam o desenvolvimento da IU.

Alguns métodos terapêuticos podem ser mais importantes não deixando de conscientizar a paciente que está com incontinência urinária precisará passar por um período de reeducação comportamental. Onde deve estabelecer ritmo miccional estipulado de hora em hora, por exemplo - visando a busca de maior intervalo de tempo entre as micções. (ROBINSON D, et al., 2014).

Pesquisas demonstram que o desconhecer o funcionamento da musculatura do assoalho pélvico pode ser um fator precipitante para IU, pois desconhecer a função muscular pode levar a obter uma hipotrofia e fraqueza (LEON M, 2001).

\section{CONCLUSÃO}

O presente estudo conclui que a IU causa grande impacto negativo na vida das mulheres modificando todo o seu comportamento diário, além de impor restrições o que compromete até mesmo seus hábitos sociais.O perfil das mulheres sugere que a prevenção e o controle da incontinência urinária sejam implementados por meio de auxilio e orientações sobre o impacto de modificações no estilo de vida, melhor controle de algumas gravidades de saúde e práticas de fortalecimento dos músculos do assoalho pélvico.

\section{REFERÊNCIAS}

1. BASAK T, KOK G, GUVENC G. Prevalence, risk factors and quality of life in Turkish women with urinary incontinence: A synthesis of the literature. International Nursing Review, 2013, 60(4), 448-460.

2. BERNARDES NO et al. Métodos de Tratamento Utilizados na Incontinência Urinária de Esforço Genuína: um Estudo Comparativo entre Cinesioterapia e Eletroestimulação Endovaginal. Revista BGO -2000; 22(1).

3. BERNARDES NO, et al. Métodos de Tratamento Utilizados na Incontinência Urinária de Esforço Genuína: um Estudo Comparativo entre Cinesioterapia e Eletroestimulação Endovaginal. Revista BGO 2000, v 22 (1),.

4. BOTELHO F, et al. Incontinência urinária feminina. Revista da Associação Portuguesa de Urologia, Lisboa, 2007;24, (1): 79-82.

5. BOTLERO R, et al. Age-specific prevalence of, and factors associated with, different types of urinary incontinence in community dwelling Australian women assessed with a validated questionnaire. Maturitas, 2008;62: 134-139.

6. BROWN JS, et al. Urinary incontinence in older women: who is at risk? Obstet Gynecol. 1996;87(5 Pt 1):715-21.

7. BUTLER RN, et al. Urinary incontinence: keys to diagnosis of the older woman. Geriatrics. 1999;54(10):22-30

8. CALVO A. O tratamento da incontinência urinária. Aten Primaria. 2002;30(5): 323-332

9. CONNOR S. Tipos de incontinência urinária, 2004.

10. CORREIAS, et al. Prevalence, treatment and known risk factors of urinary incontinence and overactive bladder in the noninstitutionalized Portuguese population. Internationa IUrogynecology Journa IPelvic Floor Dysfunction, 2009; 20, 1481-1489.

11. ELIA G, et al. Familial incontinence of urinary incontinence. Am J Obstet Gynecol. 2002; 187(1): 53-5.

12. FELDNER PC Jr, et al. Diagnóstico clínico e subsidiário da incontinência urinária Ver Bras Ginecol Obstet. 2006; 28(1): 54-62

13. FINE PFM, Clinical evaluation of women with lower urinary tract dysfunction. ClinObstetGynecol. 2004;47(1):4452.

14. GERTEN KA, et al. Impact of urinary incontinence in morbidly obese women versus women seeking urogynecologic care. Urology. 2007; 70(6):1082-5.

15. GIRÃO MJBC et al. Incontinência urinária de esforço. In: Baracat EC, Rodrigues de Lima R, editores. Guias de medicina ambulatorial e hospitalar - UNIFESP/ Escola Paulista de Medicina. São Paulo: Manole; 2005; 189-99.

16. GOMES AGP. Impacto da incontinência urinária na qualidade de vida de mulheres/ The Impacto ofurinary incontinence on quality of life of women. Rev Baiana Enferm. 2013;27(2):181-92.

17. HAN MO, et al. Abdominal obesity is associated with stress urinary incontinence in Korean women. Int Urogynecol J. 2005, 17: 35-39,

18. HIGA R, et al. Fatores de risco para incontinência urinária na mulher. Ver Esc Enferm USP, 2008, 42(1). 
19. LASSERRE, et al. Urinary incontinence in French women: Prevalence, risk factors and impact on quality of life. European Urology, 2009, 56(1), 177-83.

20. LEON M. A eficácia de um programa cinesioterapêutico para mulheres idosas com incontinência urinária. Fisioterapia Brasil 2001; 2(2): 107-15.

21. MELO BES et al. Correlação entre sinais e sintomas de incontinência urinária e autoestima em mulheres. Revista Brasileira de Geriatria e Gerontologia, Rio de Janeiro, 2012, v. 15, n. 1, p. 41- 50.

22. MESQUITA LA et al. Terapia comportamental na abordagem primária da hiperatividade do detrusor, Revista FEMINA, 2010 v. 38, № 1, janeiro.

23. MINASSIAN VA, et al. Severity of urinary incontinence and effect on quality of life in women, by incontinence type. Obstet Gynecol. 2013;121(5):1083

24. NICOLSON P, et al, It's just the worry about not being able to control it! A qualitative study of living with overactive bladder. Br J Health Psychol 2008; 13(Pt 2):343-359.

25. PENN C, et al. Assessment of urinary incontinence. J GerontolNurs. 1996;22(1):8-19.

26. PEREIRA VS, et al. Efeitos do tratamento fisioterapêutico em mulheres idosas com incontinência urinária: uma revisão sistemática. Revista Brasileira de Fisioterapia, São Carlos,2012; 16(6): 463-468.

27. RASIA J. Obesidade e qualidade de vida de mulheres pós-menopausa. [monografia]. ljuí, RS: Universidade Regional do Noroeste do Estado do Rio Grande do Sul; 2006.

28. RINCON AA. Caracterización clínica de La incontinencia urinaria y factores associados em usuarias de La Unidad de La Mujerdel Centro de Salud Familiar "Ultra estación” em la ciudad de Chillán, Chile. Rev Med Chile, 2015.

29. RIOS L A; GOMES HP. Incontinência Urinária de Esforço. Urologia Fundamental.2010.

30. ROBINSON D, et al. Urinary incontinence in the young woman: treatment plans and options available. Womens Health (Lond). 2014.

31. SANTOS PFD et al. Eletroestimulação funcional do assoalho pélvico versus terapia com os cones vaginais para o tratamento de incontinência urinária de esforço. RevBrasGinecol Obstet.; 2010; 31(9):447-52.

32. SESHAN V, Dimensions of the impact of urinary incontinence on quality of life of affected women: a review of the English literature. Int J UrolNurs. 2014;8(2): 62-70.

33. SILVA L, LOPES MHBM. Incontinência urinária em mulheres: razões da não procura por tratamento. Revista da Escola de Enfermagem da USP, São Paulo, 2009;43(1) p. 72-78.

34. SOUSA JG, et al. Avaliação da força muscular do assoalho pélvico em idosas com incontinência urinária. Revista Fisioterapia em Movimento, Curitiba, 2011; 24(1): 39-46.

35. SUBAK LL, et al. Obesity and Urinary Incontinence: Epidemiology and Clinical Research Update. The Journal of Urology. 2009; 182:S2-S7.

36. TORREALBA FCM, OLIVEIRA CR. Incontinência urinária na população feminina de idosas. Ensaios e Ciência: Ciências Biológicas, Agrárias e da Saúde, São Paulo, 2010;14, (1): 159- 175.

37. VIANA SBP, et al. Incontinência urinária e sexualidade no cotidiano de mulheres em tratamento fisioterápico: uma abordagem qualitativa. Saúde e Transformação Social, Florianópolis, 2012; 3, (4): 62-70. 